\title{
[gw22-e0094] RELATED FACTORS OF COMPLICATING DEPRESSION IN 796 PATIENTS WITH CHRONIC HEART FAILURE
}

Xianping Hua, Jinquan Hu, Zheng Cao, Yong Yang, Bin Chen, Ruixia Wu, Pingying Chen Department of Cardiology, Taihe Hospital, Hubei University of Medicine

10.1136/heartjnl-2011-300867.642

Objective To investigate the prevalence and risk factors of complicating depression in patients with chronic heart failure (CHF).

Methods The patients with CHF were continuously recruited randomly and their demographic, sociological and clinical data were collected. Hamilton Depression Scale was used to evaluate the level of depression. Stepwise logistic regression analysis was used to determined risk factors for depression.

Results The prevalence of depression was $19.22 \%$ in 796 patients with CHF. The incidence of depression was significantly higher in female patients than that in male patients $\left(30.13 \%\right.$ vs $\left.14.81 \%, x^{2}=24.65, p<0.01\right)$ and in aged patients than in middle-aged patients $\left(22.82 \%\right.$ vs $\left.14.61 \%, x^{2}=8.50, p<0.01\right)$. The prevalence of depression in patients with loss of ability to work and live was significantly higher than that in patients with work and life skills $\left(23.62 \%\right.$ vs $\left.17.16 \%, x^{2}=4.65, p<0.05\right)$ and in patients with longer course than in patients with shorter duration of illness $\left(23.48 \%\right.$ vs $\left.15.35 \%, x^{2}=5.20, p<0.05\right)$. But the prevalence of depression in smokers was significantly lower than that in non-smokers $\left(15.06 \%\right.$ vs $22.20 \%, x^{2}=6.35$, $\mathrm{p}<0.05)$. Logistic regression analysis showed the patients with the following factors such as female, the older people, longer course, and loss of ability to work and life (OR 2.47, 95\% CI 1.72 to $3.56 ; 1.72,95 \%$ CI 1.19 to $2.49 ; 1.69,95 \%$ CI 1.19 to 2.40 and $1.49,95 \%$ CI 1.03 to 2.15 , respectively) were at higher risk for depression, while smoking reduced the risk of depression (OR $0.62,95 \%$ CI 0.43 to 0.90 ).

Conclusion Around one-fifth of the CHF patients had various degrees of depression, and the factors such as female, the older people, longer course and loss of ability to work and life increased their risk of depression. 